\title{
A CLASS OF RIESZ SETS
}

\author{
DANIEL LI
}

(Communicated by Dale E. Alspach)

\begin{abstract}
Let $G$ be a metrizable compact abelian group. A subset $\Lambda$ in the dual group is said to be ergodic if every $f \in L^{\infty}(G)$ whose spectrum lies in a translate of $\Lambda$ has a unique invariant mean. It is shown that such a set is a Riesz set.
\end{abstract}

\section{INTRODUCTION}

It is well known that Rosenthal sets are Riesz sets [1, 4, 3]. A natural class of sets is lying between them; it is connected with the possibility for an element of $L^{\infty}(G)$ to have a unique invariant mean. A subset $\Lambda$ of a discrete abelian group is said to be ergodic if every $f \in L^{\infty}(G)$ whose spectrum lies in a translate of $\Lambda$ has a unique invariant mean. Katznelson [8] proved that $\mathbb{N}$, which is a Riesz set, is not an ergodic set. A few years ago, Françoise Lust-Piquard [6] showed that $\mathbb{P} \cap(5 \mathbb{Z}+3), \mathbb{P}$ being the set of primes, is an ergodic set which is not a Rosenthal set. Of course, each Rosenthal set is ergodic since continuous functions have a unique invariant mean. In view of these results, the interplay between ergodic and Riesz sets arises naturally. We prove in this paper that every ergodic set is a Riesz set.

\section{DEFINITIONS AND NOTATION}

Throughout the paper $G$ will be a metrizable compact abelian group and $\Gamma=\widehat{G}$ will be its countable discrete dual group.

As usual $\mathscr{M}_{\Lambda}, L_{\Lambda}^{1}, \mathscr{C}_{\Lambda}, L_{\Lambda}^{\infty}$ are the subspaces consisting of the elements of $\mathscr{M}(G), L^{1}(G), \mathscr{C}(G), L^{\infty}(G)$ respectively whose spectrum (i.e., the support of their Fourier transform) lies in $\Lambda \subseteq \Gamma$.

A subset $\Lambda \subseteq \Gamma$ is said to be a Riesz set if each measure $\mu \in \mathscr{M}_{\Lambda}$ is absolutely continuous (w.r.t. the Haar measure); in short, $\mathscr{M}_{\Lambda}=L_{\Lambda}^{1}$; it is said to be a Rosenthal set if each $f \in L_{\Lambda}^{\infty}$ is represented by a continuous function; in short, $L_{\Lambda}^{\infty}=\mathscr{C}_{\Lambda}$.

An invariant mean on $L^{\infty}$ is a translation-invariant continuous linear form on $L^{\infty}$ such that $\|M\|=M(1)=1$. An element $f \in L^{\infty}$ is said to be ergodic if all the invariant means take the same value on it; it is said to be totally ergodic

Received by the editors March 17, 1992.

1991 Mathematics Subject Classification. Primary 43A46; Secondary 28C10, 46B20, 04A15. 
if $\gamma f$ is ergodic for every $\gamma \in \Gamma$. For such an $f$, we have $M(\gamma f)=\hat{f}(-\gamma)$ for each invariant mean $M$.

Definition. $\Lambda \subseteq \Gamma$ is said to be an ergodic set if every $f \in L_{\Lambda}^{\infty}$ is totally ergodic.

\section{ERGODIC AND RIESZ SETS}

The main result is

Theorem 1. Let $G$ be a metrizable compact abelian group. Every ergodic set in $\Gamma=\widehat{G}$ is a Riesz set.

Proof. For every $\mu \in \mathscr{M}_{\Lambda}$, we write $\mu=\mu_{a}+\mu_{s}$, its Lebesgue-Radon-Nikodým decomposition. For every $f \in L^{\infty}$, we have $\mu * f \in L_{\Lambda}^{\infty}$ and so $\mu * f$ is totally ergodic. Since $\mu_{a} * f$ is totally ergodic, as a continuous function, we obtain that $\mu_{s} * f$ is totally ergodic. The following proposition allows us to conclude that $\mu_{s}=0$.

Proposition 2. Let $G$ be a metrizable compact abelian group. For every singular measure $\sigma \neq 0$, there is an $f \in L^{\infty}$ such that $\sigma * f$ is not totally ergodic.

Proof. First, let $\mu$ be a singular measure such that $\theta=\mu(G) \neq 0$.

Since the measure $\nu=|\mu|$ is singular, there is a Borel set $B$ such that $m(B)=0$ ( $m$ being the Haar measure on $G$ ) and such that $\nu(B)=\|\nu\|$. It will be convenient to take $B$ symmetric.

Let $D$ be a countable dense subgroup of $G$. We set $A=B+D$; since $D$ is countable, we have $m(A)=0$, and since $B \subseteq A$, we have $\nu(A)=\|\nu\|$.

Let now $\Omega$ be an open set of $G$ containing $A$ and such that $m(\Omega) \leq 1 / 4$.

We are going to show that $\mu * \mathbf{1}_{\Omega}$ is not ergodic. For this, we shall use the fact that an element $f \in L^{\infty}$ is ergodic if and only if there is a constant function in the norm-closed convex hull of the orbit of $f$ by $G[5,8]$.

Let $\lambda_{1}, \ldots, \lambda_{n} \geq 0$ with $\sum_{i=1}^{n} \lambda_{i}=1$ and $x_{1}, \ldots, x_{n} \in G$ be given. For each $i \in\{1, \ldots, n\}$, the function $\varphi_{i}$ defined by

$$
\varphi_{i}(x)=\left(\mathbf{1}_{\left(\Omega+x_{i}\right)} * \nu\right)(x)=\left(\mathbb{1}_{\Omega} * \nu\right)_{x_{i}}(x)=\nu\left(\Omega+x-x_{i}\right)
$$

is l.s.c.; therefore the set

$$
U_{i}=\left\{x \in G ; \nu\left(\Omega+x-x_{i}\right)>\|\nu\|-|\theta| / 4\right\}
$$

is open. Moreover, it is dense since it contains $D+x_{i}$. Then, $U=\bigcap_{i=1}^{n} U_{i}$ is also a dense open set, so $m(U)>0$.

Moreover, for each $x \in U$, we have

$$
\forall i \in\{1, \ldots, n\} \quad \nu\left(\Omega+x-x_{i}\right)>\|\nu\|-|\theta| / 4 .
$$

We have then, for each $x \in U$,

$$
\begin{aligned}
\left|\sum_{i=1}^{n} \lambda_{i} \mu\left(\Omega+x-x_{i}\right)-\mu(\Omega)\right| & =\left|\sum_{i=1}^{n} \lambda_{i}\left[\mu\left(\Omega+x-x_{i}\right)-\mu(\Omega)\right]\right| \\
& \leq \sum_{i=1}^{n} \lambda_{i} \nu\left(\left(\Omega+x-x_{i}\right) \Delta \Omega\right)<\frac{|\theta|}{4}
\end{aligned}
$$


and so, since $\mu(\Omega)=\mu(G)=\theta$,

$$
\left|\sum_{i=1}^{n} \lambda_{i} \mu\left(\Omega+x-x_{i}\right)\right|>\frac{3|\theta|}{4} .
$$

Therefore,

$$
\left\|\sum_{i=1}^{n} \lambda_{i}\left(\mathbb{1}_{\Omega} * \mu\right)_{x_{i}}\right\|_{\infty}>\frac{3|\theta|}{4} .
$$

Hence, there is no constant function in the norm-closed convex hull of $\left\{\left(1_{\Omega} * \mu\right)_{x} ; x \in G\right\}$, since if there is one, namely, $C 1$, we do have by the above calculations $C \geq 3|\theta| / 4$; but, on the other hand, we also have

$$
C=\int_{G}\left(\mathbb{1}_{\Omega} * \mu\right) d m=\mu(G) m(\Omega)
$$

and so $|C| \leq|\theta| / 4$.

This proves that $\mathbf{1}_{\Omega} * \mu$ is not ergodic.

To finish the proof, let $\gamma \in \Gamma$ be such that $\hat{\sigma}(\gamma) \neq 0$. Setting $\mu=\bar{\gamma} \sigma$, we have $\mu(G) \neq 0$, and then the above proof shows that

$$
\bar{\gamma}\left[\sigma *\left(\gamma \mathbf{1}_{\Omega}\right)\right]=(\bar{\gamma} \sigma) * \mathbf{1}_{\Omega}
$$

is not ergodic; therefore, $\sigma *\left(\gamma \mathbf{1}_{\Omega}\right)$ is not totally ergodic.

Corollary 3. Let $\Gamma$ be a countable (infinite) discrete abelian group. The set $\mathscr{E} r g(\Gamma)$ of all the ergodic sets in $\Gamma$ is a coanalytic subset of $\mathscr{P}(\Gamma)$ which is not a Borel subset.

Proof. From [2, Theorem 2] and Theorem 1 above, $\mathscr{E} r g(\Gamma)$ is not an analytic subset of $\mathscr{P}(\Gamma)$. It is then enough to show that it is coanalytic.

An element $f \in L^{\infty}(G)$ is ergodic if and only if for every $k \geq 1$ there is an integer $n$ and elements $x_{1}, \ldots, x_{n}$ in the group $G$ such that

$$
\left\|\frac{1}{n} \sum_{i=1}^{n} f_{x_{i}}-\hat{f}(0) \mathbf{1}\right\|_{\infty} \leq \frac{1}{k} ;
$$

hence, the set $E(G)$ of all the totally ergodic elements of $L^{\infty}(G)$ can be written as

where

$$
E(G)=\bigcap_{\gamma \in \Gamma} \bigcap_{k \geq 1} \bigcup_{n \geq 1} \bigcup_{M \geq 1} F_{k, n, M},
$$

$$
F_{k, n, M}=\left\{f \in L^{\infty}(G) ;\|f\|_{\infty} \leq M \text { and } \exists x_{1}, \ldots, x_{n} \in G:(*)\right\} .
$$

$F_{k, n, M}$ is $w^{*}$-compact, as the projection of the compact

$$
\left\{\left(x_{1}, \ldots, x_{n}, f\right) \in G^{n} \times\left(B_{L^{\infty}(G)}(0, M), w^{*}\right) ;(*)\right\} .
$$

This shows that $E(G)$ is a $K_{\sigma \delta}$. To finish the proof, it suffices to check that $\mathscr{P}(\Gamma) \backslash \mathscr{E} r g(\Gamma)$ is the projection of the $G_{\delta \sigma}$ :

$$
\left\{(\Lambda, f) \in \mathscr{P}(\Gamma) \times\left(L^{\infty}(G), w^{*}\right) ; \hat{f}=0 \text { on } \Lambda^{c} \text { and } f \in L^{\infty}(G) \backslash E(G)\right\}
$$

and so is analytic. 
Remark. As was recalled in the introduction, every Rosenthal set is a Riesz set. More precisely, we have the following implications:

$$
\text { (i) } \Rightarrow \text { (ii) } \Rightarrow \text { (iii) } \Rightarrow \text { (iv) } \Rightarrow \text { (v) }
$$

between the properties:

(i) $\Lambda$ is a Rosenthal set;

(ii) the space $L^{1} / L_{\Gamma \backslash(-\Lambda)}^{1}$ contains no isomorphic copy of $l_{1}$ (since its dual space $L_{\Lambda}^{\infty}$ has the Radon-Nikodým Property);

(iii) every element of $L_{\Lambda}^{\infty}$ is represented by a Riemann-integrable function (by [7, Corollary IV.4]);

(iv) $\Lambda$ is an ergodic set (since every Riemann-integrable function is totally ergodic; see, for instance, [8] or [9]);

(v) $\Lambda$ is a Riesz set (by Theorem 1).

\section{ACKNOWLEDGMENT}

I am grateful to Françoise Lust-Piquard, Gilles Godefroy, and Michel Talagrand for fruitful conversations.

\section{REFERENCES}

1. R. E. Dressler and L. Pigno, Rosenthal sets and Riesz sets, Duke Math. J. 41 (1974), 675-677.

2. G. Godefroy, On coanalytic families of sets in harmonic analysis, Illinois J. Math. 35 (1991), 241-249.

3. F. Lust, Ensembles de Rosenthal et ensembles de Riesz, C. R. Acad. Sci. Paris 282 (1976), 833-835.

4. F. Lust-Piquard, Propriétés géométriques des sous-espaces invariants par translation de $L^{1}(G)$ et $C(G)$, Sém. Géom. Esp. Banach École Polytechn., Mimeog. Ecole Polytechnique, Palaiseau, France, 1977-78, exposé no. 26.

5. __ Eléments ergodiques et totalement ergodiques dans $L^{\infty}(G)$, Studia Math. 69 (1981), 191-225.

6. B Bohr local properties of $C_{\Lambda}(\mathbb{T})$, Colloq. Math. 58 (1989), 29-38.

7. F. Lust-Piquard and W. Schachermayer, Functions in $L^{\infty}(G)$ and associated convolutions operators, Studia Math. 93 (1989), 109-136.

8. L. A. Rubel and A. L. Shields, Invariant subspaces of $L^{\infty}$ and $H^{\infty}$, J. Reine Angew. Math. 272 (1975), 32-44.

9. M. Talagrand, Some functions with a unique invariant mean, Proc. Amer. Math. Soc. 82 (1981), 253-256.

Analyse Harmonique, Universite Paris-Sud, Bat.425, 91405 Orsay Cedex, France

E-mail address: li@anh.matups.fr

Equipe d'Analyse, Universite Paris VI, tour 46-0 4Eme etage, 75252 Paris Cedex, France E-mail address: li@matups.matups.fr 